\title{
Youla-Kucera adaptive feedback disturbance rejection in the presence of plant uncertainties
}

\author{
Bernard Vau and Ioan Doré Landau
}

\begin{abstract}
The stability of an adaptive disturbance rejection scheme based on the Youla-Kucera parameterization is investigated, in case of an uncertain plant model is used in the synthesis of the central controller. It is shown that stability is guaranteed provided that two conditions are satisfied at the same time: the first one is linked to the internal model principle, and the second one depends on the closed-loop poles location. For some uncertainties, these constraints cannot be met simultaneously with the minimal Q-filter. That leads to propose an over-parametrized Youla-Kucera filter, in order to relax the said conditions. Simulations on relevant examples illustrate the procedure for stabilizing the Youla-Kucera adaptive rejection scheme, in the presence of plant model uncertainties.
\end{abstract}

\section{INTRODUCTION}

In the last twenty years, the issue of adaptive rejection of unknown disturbances has received a significant interest in the field of control. Most of the proposed algorithms are based on the Youla-Kucera parameterization (or Qparameterization) [1], that allows for the tuning of a compensator within the class of all-stabilizing controllers. The first contribution suggesting to blend a robust controller with an adaptive structure established on the Q-filter seems to have been reported in [9], and a successful adaptive rejection of disturbances resulting from an unstable exogenous system is mentioned in [12]. In the field of active noise control, a first reference seems to be [2], [3] and a more recent reference is [8]. In the field of vibration control a first reference seems to be [6]. In all these papers, the system to be controlled is assumed to be perfectly known, and under this assumption these algorithms do not require a specific stability condition, except that the closed-loop including the central controller must be stable. However in many situations despite excellent experimental identification techniques, the plant model is subject to changes during operation and therefore the plant model uncertainties have to be taken into account. In order to address the issue of plant model uncertainties, Kinney and Callafon proposed an adaptive scheme called REACT [5] that aims at rejecting unknown disturbances acting on a uncertain system. The uncertainties are represented by means of the dual Youla-Kucera parameterization [1] that had been previously used in the context of identification (see for example [11]). In the REACT scheme, conditions about

This work was not supported by any organization

B.Vau is with SATIE, école normale supérieure de Paris-Saclay, 94230 Cachan, France

and with IXBLUE, 12 avenue des coquelicots 94385 Bonneuil-sur-Marne, France bernard.vaudsatie.ens-cachan. fr

I.D. Landau is with the Univ. Grenoble Alpes, CNRS, Grenoble INP, GIPSA-lab, 38000 Genoble France ioan. dore. landaulgipsa-lab.grenoble-inp. fr stability are provided by means of the small gain theorem, employed in the case of static controller coefficients, but no stability analysis of the adaptive structure is established. In REACT, the error signal is directly the system output. This output can be expressed in an affine manner from the Q-filter when it has static coefficients, but this affine relation is lost in case of time-varying gains in the Q-filter, because of the non-commutativity of time varying transfer operators. It is probably one reason that compelled the authors of [5] to use regularization in the recursive estimation algorithm (LMS), which prevents the estimated parameters from a possible divergence. Contrary to REACT, the structure presented in [6], has an adaptation error being in any case affine with respect to the estimated parameters, and for this reason the stability analysis is more tractable. We propose here to establish stability conditions of the latter scheme that we call the adaptive disturbance rejection algorithm, when the plant model is uncertain. The uncertainties are expressed with the dual Youla-Kucera parameterization, and we show that stability is guaranteed provided two conditions are simultaneously satisfied. The first one is a consequence of the internal model principle of Francis and Wohnam [4], expressed by means of a Bezout equation, which reveals to be independent with respect to uncertainties. The second condition is linked to the location of the closed-loop poles, that are no longer invariant with respect to the (finite impulse response) Q-filter parameters, if the actual system differs from the plant model, contrary to what happens when the plant and the model match perfectly. In some situations, the minimal solution of the said Bezout equation, may not lead to a stable closed-loop, and consequently the convergence cannot be obtained. In order to overcome this issue, a solution consists in augmenting the order of the Q-filter until a solution of the Bezout equation allows to fulfil the stability condition. While this idea has been suggested in [13], pp. 4849 , this approach has never been worked in detail to establish a clear procedure.

The main contributions of this paper are: 1) A clear formulation of the stability issues in adaptive rejection of disturbances using Youla-Kucera parameterization when taking into account the plant model uncertainties, 2) A stability analysis in the presence of plant model uncertainties when using a minimal order Q-filter, 3) Development of a procedure for using overparametrized Q-filters in order to ensure stability in the presence of plant model uncertainties, 4) Illustration of the procedures developed on relevant simulation examples.

The paper is organized as follows: Section II proposes a 
description of the uncertain plant. In section III a stability analysis of the adaptive disturbance rejection algorithm is provided. Section IV shows the advantage of an overparametrization of the Q-filter, and finally in section V simulations on relevant examples illustrate the procedure for stabilizing the Youla-Kucera adaptive rejection scheme, in the presence of plant model uncertainties.

\section{DESCRIPTION OF THE UNCERTAIN SYSTEM}

Let us consider the nominal system $G_{o}\left(q^{-1}\right), q^{-1}$ being the shift backward operator

$$
G_{o}\left(q^{-1}\right)=\frac{B_{o}\left(q^{-1}\right)}{A_{o}\left(q^{-1}\right)}
$$

$u(t)$ and $y(t)$ are the system input, and output respectively, and set $d(t)$ an unknown harmonic disturbance acting additively on the system output such that

$$
d(t)=\frac{N_{d}\left(q^{-1}\right)}{D_{d}\left(q^{-1}\right)} \delta(t)
$$

where $\delta(t)$ is the Dirac symbol.

This nominal system is used in the synthesis of a central controller $C_{o}\left(q^{-1}\right)$ with

$$
C_{o}\left(q^{-1}\right)=\frac{R_{o}\left(q^{-1}\right)}{S_{o}\left(q^{-1}\right)}
$$

and a Youla-Kucera filter (or Q-filter) is added to the initial controller structure,

$$
Q\left(q^{-1}\right)=\frac{B_{Q}\left(q^{-1}\right)}{A_{Q}\left(q^{-1}\right)}
$$

In the following, the Q-filter is assumed to be finite impulse response filter, thus $A_{Q}\left(q^{-1}\right)=1$ and

$$
B_{Q}\left(q^{-1}\right)=b_{0}^{Q}+b_{1}^{Q} q^{-1}+\cdots b_{n b}^{Q} q^{-n b}
$$

where $n_{b}$ is the degree of $B_{Q}\left(q^{-1)}\right.$.

Consequently, the relation from the system output to the control signal is

$$
u(t)=-\frac{R_{o}\left(q^{-1}\right)+B_{Q}\left(q^{-1}\right) A_{o}\left(q^{-1}\right)}{S_{o}\left(q^{-1}\right)-B_{Q}\left(q^{-1}\right) B_{o}\left(q^{-1}\right)} y(t)
$$

The system model has parametric uncertainties, and we propose to express them by means of the Dual-Youla parameterization, as in [5]. Let $Q_{D}\left(q^{-1}\right)$ be this Dual-Youla filter

$$
Q_{D}\left(q^{-1}\right)=\frac{\Delta\left(q^{-1}\right)}{\Gamma\left(q^{-1}\right)}
$$

The numerator $\Delta\left(q^{-1}\right)$ does not include any direct transmission, and $\Gamma\left(q^{-1}\right)$ is monic. Then the uncertain system is given by

$$
G\left(q^{-1}\right)=\frac{B\left(q^{-1}\right)}{A\left(q^{-1}\right)}=\frac{\Gamma\left(q^{-1}\right) B_{o}\left(q^{-1}\right)+\Delta\left(q^{-1}\right) S_{o}\left(q^{-1}\right)}{\Gamma\left(q^{-1}\right) A_{o}\left(q^{-1}\right)-\Delta\left(q^{-1}\right) R_{o}\left(q^{-1}\right)}
$$

The relation from the uncertain system input to its output is

$$
y(t)=G\left(q^{-1}\right) u(t)+d(t)
$$

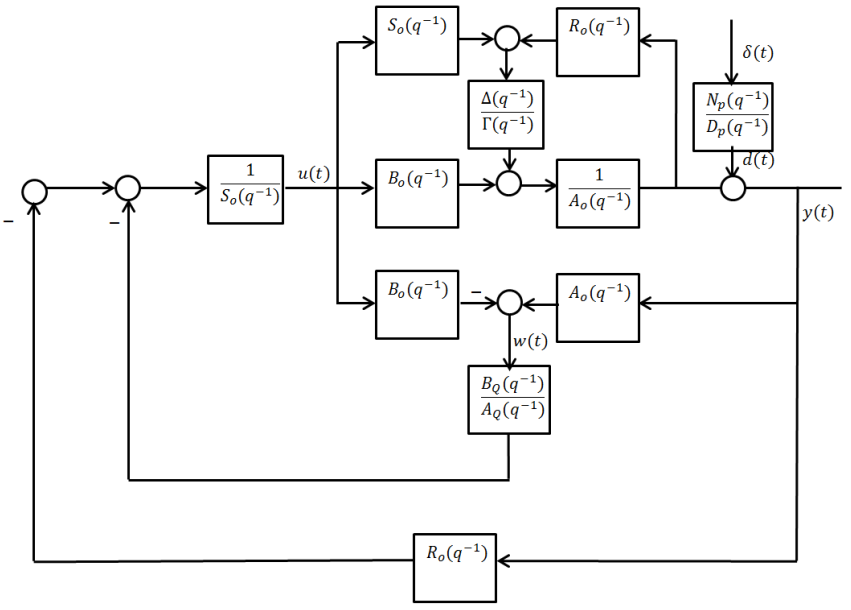

Fig. 1. Global block diagram of the uncertain system and the controller including the Q-filter

and the global structure representing the uncertain system with the controller and the Q-filter is provided in Fig. 1. In the presence of the central controller, the closed-loop poles are defined by the roots of

$$
P_{o}\left(q^{-1}\right)=A_{o}\left(q^{-1}\right) S_{o}\left(q^{-1}\right)+B_{o}\left(q^{-1}\right) R_{o}\left(q^{-1}\right)
$$

The description of uncertainties with the Dual Youla-Kucera parameterization is the most general, provided $Q_{D}\left(q^{-1}\right)$ is an infinite impulse response filter. From (7), one checks immediately that

$$
\frac{\Delta\left(q^{-1}\right)}{\Gamma\left(q^{-1}\right)}=\frac{B\left(q^{-1}\right) A_{o}\left(q^{-1}\right)-A\left(q^{-1}\right) B_{o}\left(q^{-1}\right)}{A\left(q^{-1}\right) S_{o}\left(q^{-1}\right)+B\left(q^{-1}\right) R_{o}\left(q^{-1}\right)}
$$

Note that $\Gamma\left(q^{-1}\right)$ is nothing but the characteristic polynomial of the closed-loop including the uncertain system and the central controller. Since it is reasonable to assume that the uncertain system is stabilized by the central controller, this is equivalent for $\Gamma\left(q^{-1}\right)$ to be a stability polynomial. From (5) and (7), the computation of the direct sensitivity function from $d(t)$ to $y(t)$ can be carried out. One obtains

$$
\begin{aligned}
y(t)=\frac{\Gamma A_{0}\left(q^{-1}\right)-\Delta\left(q^{-1}\right) R_{0}\left(q^{-1}\right)}{P_{0}\left(q^{-1}\right)} \times \\
\frac{\left(S_{0}\left(q^{-1}\right)-B_{Q}\left(q^{-1}\right) B_{0}\left(q^{-1}\right)\right)}{\Gamma\left(q^{-1}\right)+B_{Q}\left(q^{-1}\right) \Delta\left(q^{-1}\right)} d(t)
\end{aligned}
$$

it appears immediately that in case of an uncertain system, some poles of the direct sensitivity function are no longer invariant, and they depend on $B_{Q}\left(q^{-1}\right)$, contrary to the case without system uncertainty.

\section{Stability analysis of the adaptive DISTURBANCE REJECTION ALGORITHM}

The stability analysis in the presence of plant model uncertainties will be achieved in the case of a minimal order 
Q-filter. In an on-line implementation, $B_{Q}\left(q^{-1}\right)$ is replaced by $\hat{B}_{Q}\left(q^{-1}, t\right)=\hat{b}_{0}^{Q}(t)+\cdots \hat{b}_{n b}^{Q}(t) q^{-1}$ that results from an estimation described below. If $\hat{B}_{Q}\left(q^{-1}, t\right)$ has constant coefficients (11) becomes

$$
\begin{aligned}
y(t)=\frac{\Gamma A_{0}\left(q^{-1}\right)-\Delta\left(q^{-1}\right) R_{0}\left(q^{-1}\right)}{P_{0}\left(q^{-1}\right)} \times \\
\frac{\left(S_{0}\left(q^{-1}\right)-\hat{B}_{Q}\left(q^{-1}\right) B_{0}\left(q^{-1}\right)\right)}{\Gamma\left(q^{-1}\right)+\hat{B}_{Q}\left(q^{-1}\right) \Delta\left(q^{-1}\right)} d(t)
\end{aligned}
$$

The signal $w(t)$ in Fig.1 has the expression

$$
w(t)=-B_{o}\left(q^{-1}\right) u(t)+A_{o}\left(q^{-1}\right) y(t)
$$

The following property holds

Property 1: In case of model uncertainty expressed with the Dual-Youla parameterization as in (7), one has

$$
w(t)=\frac{\Gamma\left(q^{-1}\right) A o\left(q^{-1}\right)-\Delta\left(q^{-1}\right) R_{o}\left(q^{-1}\right)}{\Gamma\left(q^{-1}\right)+\Delta\left(q^{-1}\right) \hat{B}_{Q}\left(q^{-1}\right)} d(t)
$$

Proof: From (13) $w(t)=-B_{o}\left(q^{-1}\right) u(t)+$ $A_{o}\left(q^{-1}\right) y(t)$, and then $\Gamma w(t)=\left(\Gamma A_{o}-\Delta R_{o}\right) d(t)+$ $\Delta\left[S_{o} u(t)+R_{o} y(t)\right]$. But owing to the expression of the controller including the Q-filter, one obtains $\Delta\left[S_{o} u(t)+\right.$ $\left.R_{o} y(t)\right]=-\frac{\Delta \hat{B}_{Q} P_{o}}{S_{o}-\hat{B}_{Q} B_{o}} y(t)$. Thus $\left(\Gamma A_{o}-\Delta R_{o}\right) d(t)=$ $\Gamma w(t)+\frac{\Delta \hat{B}_{Q} P_{o}}{S_{o}-\hat{B}_{Q} B_{o}} y(t)$. Owing to the expression of the direct sensitivity function given in (12), one can write the equality $\left(\Gamma A_{o}-\Delta R_{o}\right) d(t)=\Gamma w(t)+\frac{\Delta \hat{B}_{Q}\left(\Gamma A_{o}-\Delta R_{o}\right)}{\Gamma+\hat{B}_{Q} \Delta}$ hence the final result $w(t+1)=\frac{\left(\Gamma A_{o}-\Delta R_{o}\right)}{\left(\Gamma+\Delta \hat{B}_{Q}\right.} d(t+1)$.

By combining (12) and (14), one obtains

$$
y(t)=\left(\frac{S_{o}\left(q^{-1}\right)}{P_{o}\left(q^{-1}\right)}-\frac{\hat{B}_{Q}\left(q^{-1}, t\right) B_{o}\left(q^{-1}\right)}{P_{o}\left(q^{-1}\right)}\right) w(t)
$$

But in the adaptive rejection algorithm proposed in [6], an (a-posteriori) adaptation error $\varepsilon(t+1)$ is defined as

$\varepsilon(t+1)=\left(\frac{S_{o}\left(q^{-1}\right)}{P_{o}\left(q^{-1}\right)}-\hat{B}_{Q}\left(q^{-1}, t+1\right) \frac{B_{o}\left(q^{-1}\right)}{P_{o}\left(q^{-1}\right)}\right) w(t+1)$

Similarly an a-priori adaptation error $\varepsilon^{o}(t+1)$ is

$$
\varepsilon^{o}(t+1)=\left(\frac{S_{o}\left(q^{-1}\right)}{P_{o}\left(q^{-1}\right)}-\hat{B}_{Q}\left(q^{-1}, t\right) \frac{B_{o}\left(q^{-1}\right)}{P_{o}\left(q^{-1}\right)}\right) w(t+1)
$$

Now, owing to (11), it is clear that in order to reject asymptotically the output disturbance, the internal model principle [4] can be expressed with the following Bezout equation

$$
D_{p}\left(q^{-1}\right) S^{\prime}\left(q^{-1}\right)+B_{Q}\left(q^{-1}\right) B_{o}\left(q^{-1}\right)=S_{o}\left(q^{-1}\right)
$$

where $S^{\prime}\left(q^{-1}\right)$ et $B_{Q}\left(q^{-1}\right)$ are the unknown terms. The minimal solution of this equation is such that $n_{b}=\operatorname{deg}\left(B_{Q}\right)=$ $\operatorname{deg}\left(D_{p}\right)-1$. In this section, we are interested only by this minimal solution. From (16) and (18), the adaptation error can be written

$$
\begin{array}{r}
\varepsilon(t+1)=\left(B_{Q}\left(q^{-1}\right)-\hat{B}_{Q}\left(q^{-1}, t+1\right)\right) \frac{B_{o}^{*}\left(q^{-1}\right)}{P_{o}\left(q^{-1}\right)} w(t) \\
+x(t+1)
\end{array}
$$

where $x(t+1)=\frac{D_{p}\left(q^{-1}\right) S^{\prime}\left(q^{-1}\right)}{P_{o}\left(q^{-1}\right)} w(t+1)$ is a signal that tends rapidly towards 0 . By comparison with [6], one notices that the adaptation error remains unchanged even if the model is uncertain, the only difference lying in the expression of $w(t)$.

Set

$$
w_{2}(t)=\frac{B^{*}\left(q^{-1}\right)}{P_{0}\left(q^{-1}\right)} w(t)
$$

the adaptation error can be put under the form

$$
\varepsilon(t+1)=H\left(q^{-1}\right)(\theta-\hat{\theta}(t+1))^{T} \phi(t)
$$

where

$$
\begin{gathered}
\phi^{T}(t)=\left[w_{2}(t) \cdots w_{2}(t-n b)\right] \\
\theta^{T}=\left[b_{0}^{Q} \cdots b_{n b}^{Q}\right] \\
\hat{\theta}^{T}(t)=\left[\hat{b}_{0}^{Q}(t) \cdots \hat{b}_{n b}^{Q}(t)\right]
\end{gathered}
$$

and

$$
H\left(q^{-1}\right)=1
$$

The expression of the adaptation error in (21) is often encountered in adaptive control, and it is well known (see theorem 3.2 in [7]) that a passivity condition upon the transfer function $H\left(q^{-1}\right)$ exists. This condition is obviously satisfied here since $H\left(q^{-1}\right)=1$, therefore no passivity constraint prevents the adaptive rejection algorithm from converging even in case of system uncertainties.

The coefficients of vector $\hat{\theta}(t)$ are estimated by means of the so-called parameter adaptation algorithm (see [7])

$$
\widehat{\theta}(t+1)=\widehat{\theta}(t)+F(t) \phi(t) \varepsilon(t+1)
$$

$$
F(t+1)=\frac{1}{\lambda_{1}(t)}\left[F(t)-\frac{F(t) \phi(t) \phi^{T}(t) F(t)}{\frac{\lambda_{1}(t)}{\lambda_{2}(t)}+\phi^{T}(t) F(t) \phi(t)}\right]
$$

where $F(t)$ is the adaptation gain (positive definite matrix), and $0<\lambda_{1}(t) \leq 1,0 \leq \lambda_{2}(t)<2$ are the forgetting factors. The a-posteriori adaptation error is computed from the apriori adaptation error expression (17), and from

$$
\varepsilon(t+1)=\frac{\varepsilon^{o}(t+1)}{1+\phi^{T}(t) F(t) \phi(t)}
$$

Notice that from the expression of the direct sensitivity function (11), the internal model principle leads to an unique Bezout equation (18), invariant under system uncertainties: $B_{Q}\left(q^{-1}\right)$ depends on $S_{o}\left(q^{-1}\right), D_{p}\left(q^{-1}\right)$, but not on $\Gamma\left(q^{-1}\right)$ and $\Delta\left(q^{-1}\right)$. However as said at the end of section II, the closed-loop polynomial becomes equal to 
$P_{o}\left(q^{-1}\right)\left(\Gamma\left(q^{-1}\right)+B_{Q}\left(q^{-1}\right) \Delta\left(q^{-1}\right)\right)$. And this polynomial can possibly exhibit some zeros outside the unit circle, which jeopardize the closed-loop stability.

$\phi(t)$ will be finite if and only if all the zeros of the polynomial $\Gamma\left(q^{-1}\right)+\Delta\left(q^{-1}\right) \hat{B}_{Q}\left(q^{-1}\right)$ remain inside the unit circle. On the other hand it is necessary for $\hat{B}_{Q}$ to tend towards $B_{Q}\left(q^{-1}\right)$ which must satisfy (18). In some situation an incompatibility may arise from these two constraints especially if the uncertainties are large. In this case the algorithm either does not converge or it tends towards values which leads to an unstable closed-loop. To overcome this difficulty, one solution is to overparametrize the Q-filter as it will be shown next.

\section{OVERPARAMETERIZATION OF THE YOULA BLOCK}

In this section, we call $\bar{B}_{Q}\left(q^{-1}\right)$ the minimal solution of the Bezout equation corresponding to the internal model condition

$$
D_{p}\left(q^{-1}\right) S^{\prime}\left(q^{-1}\right)+\bar{B}_{Q}\left(q^{-1}\right) B_{o}\left(q^{-1}\right)=S_{o}\left(q^{-1}\right)
$$

with $\operatorname{deg}\left(\bar{B}_{Q}\right)=\operatorname{deg}\left(D_{p}\right)-1$. Any non-minimal solution $B_{Q}\left(q^{-1}\right)$ in (18) can be obtained from $\bar{B}_{Q}\left(q^{-1}\right)$. As a matter of fact (24) is equivalent to

$$
\begin{aligned}
& D_{p}\left(q^{-1}\right)\left(S^{\prime}\left(q^{-1}\right)-V\left(q^{-1}\right) B_{o}\left(q^{-1}\right)\right)+ \\
& \left(\bar{B}_{Q}\left(q^{-1}\right)+V\left(q^{-1}\right) D_{p}\left(q^{-1}\right)\right) B_{o}\left(q^{-1}\right)=S_{o}\left(q^{-1}\right)
\end{aligned}
$$

where $V\left(q^{-1}\right)$ is a polynomial which parametrizes (25). The set of solutions $B_{Q}\left(q^{-1}\right)$ in (18) is given by the expression $B_{Q}\left(q^{-1}\right)=\bar{B}_{Q}\left(q^{-1}\right)+V\left(q^{-1}\right) D_{p}\left(q^{-1}\right)$. Furthermore if $\hat{B}\left(q^{-1}\right)$ is overparametrized, the polynomial $V\left(q^{-1}\right)$ must allow for $\hat{B}\left(q^{-1}\right)$ to tend towards $\bar{B}_{Q}\left(q^{-1}\right)+V\left(q^{-1}\right) D_{p}\left(q^{-1}\right)$.

Property 2: In case of an uncertain system described by means of the Dual-Youla parameterization, controlled by the adaptive disturbance rejection algorithm, the closed-loop is asymptotically stable, and the regressor $\phi(t)$ remains bounded if there exists at least one polynomial $V\left(q^{-1}\right)$ such that

$$
\Gamma\left(q^{-1}\right)+\Delta\left(q^{-1}\right) \bar{B}_{Q}\left(q^{-1}\right)+\Delta\left(q^{-1}\right) V\left(q^{-1}\right) D_{p}\left(q^{-1}\right)
$$

has all its zeros in the unit circle, with $\bar{B}_{Q}\left(q^{-1}\right)$ the minimal solution of the Bezout equation

$$
D_{p}\left(q^{-1}\right) S^{\prime}\left(q^{-1}\right)+\bar{B}_{Q}\left(q^{-1}\right) B_{o}\left(q^{-1}\right)=S_{o}\left(q^{-1}\right)
$$

Proof: $\quad$ From (14) and (20), a sufficient condition for $\phi(t)$ to remain bounded is that $\Gamma\left(q^{-1}\right)+\Delta\left(q^{-1}\right) B_{Q}\left(q^{-1}\right)$ be a stability polynomial. Since $B_{Q}\left(q^{-1}\right)=\bar{B}\left(q^{-1}\right)+V\left(q^{-1}\right) D_{p}\left(q^{-1}\right)$, a sufficient condition for $\phi(t)$ to be bounded is that $\Gamma\left(q^{-1}\right)+\Delta\left(q^{-1}\right) \bar{B}_{Q}\left(q^{-1}\right)+\Delta\left(q^{-1}\right) V\left(q^{-1}\right) D_{p}\left(q^{-1}\right)$ has all its zeros inside the unit circle. On the other hand, according to (24), the internal model principle is satisfied provided $D_{p}\left(q^{-1}\right) S^{\prime}\left(q^{-1}\right)+\bar{B}_{Q}\left(q^{-1}\right) B_{o}\left(q^{-1}\right)=S_{o}\left(q^{-1}\right)$.

For a given order of $V\left(q^{-1}\right)$, there may be no coefficient such that the condition of property 2 is satisfied. In this situation, the only remaining solution consists in augmenting the order of $V\left(q^{-1}\right)$, and consequently the order of $B_{Q}\left(q^{-1}\right)$.

\section{Simulation EXAMPles}

Let us consider the following system $G_{o}\left(q^{-1}\right)=\frac{B_{o}\left(q^{-1}\right)}{A_{o}\left(q^{-1}\right)}$, and the controller $C_{o}\left(q^{-1}\right)=\frac{R_{o}\left(q^{-1}\right)}{S_{o}\left(q^{-1}\right)}$ (central controller), where

$$
\begin{gathered}
B_{o}\left(q^{-1}\right)=0.5 q^{-1}-0.5 q^{-2} \\
A_{o}\left(q^{-1}\right)=1-1.45 q^{-1}+0.475 q^{-2} \\
R_{o}\left(q^{-1}\right)=0.15-0.14 q^{-1} \\
S_{o}\left(q^{-1}\right)=1-0.74 q^{-1}
\end{gathered}
$$

In a first set of simulation (first configuration) the expression of $\Gamma\left(q^{-1}\right)$ and $\Delta\left(q^{-1}\right)$ are

$$
\begin{gathered}
\Gamma\left(q^{-1}\right)=1-1.4 q^{-1}+0.45 q^{-2} \\
\Delta\left(q^{-1}\right)=0.09 q^{-1}-0.09 q^{-2}
\end{gathered}
$$

The uncertain system and the nominal one are represented in Fig. 2

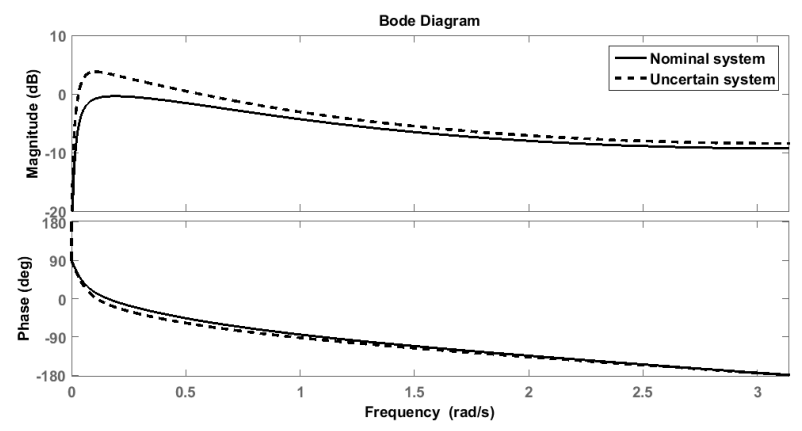

Fig. 2. Uncertain and nominal systems, (first uncertainty configuration)

The sampling period is equal to 1 , and one assumes that an harmonic (single frequency) output disturbance acts upon the system, with a pulsation equal to $\omega=0.2$. Consequently

$$
D_{p}\left(q^{-1}\right)=1-1.96 q^{-1}+q^{-2}
$$

Under these assumptions the minimal order polynomial $\bar{B}_{Q}\left(q^{-1}\right)$ is $\bar{B}_{Q}\left(q^{-1}\right)=-8.56+11 q^{-1}$. If there is no uncertainty, the condition of persistent excitation is satisfied (see [6]), leading not only to stability but to parameters convergence too. One can check easily that $\Gamma\left(q^{-1}\right)+$ $\bar{B}_{Q}\left(q^{-1}\right) \Delta\left(q^{-1}\right)$ has not all its zeros inside the unit circle, and a simulation of the adaptive rejection algorithm applied to this system shows that convergence is not obtained: At first the coefficients of $\hat{B}_{Q}\left(q^{-1}\right)$ tend toward those of $\bar{B}_{Q}\left(q^{-1}\right)$, 

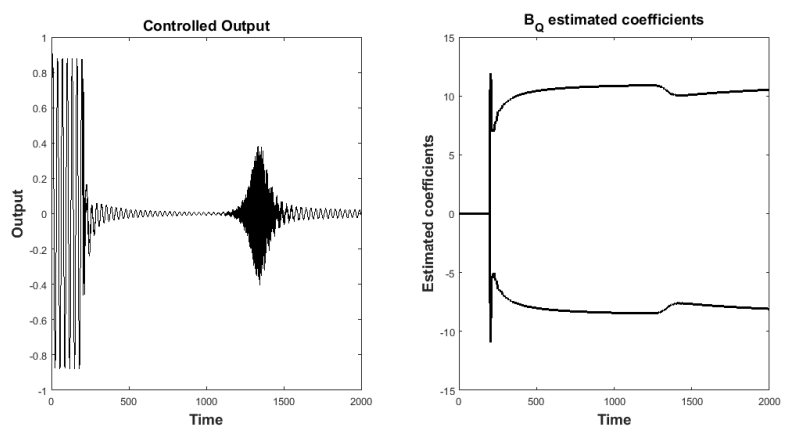

Fig. 3. Adaptive rejection with a first order $\hat{B}_{Q}\left(q^{-1}\right)$ (first uncertainty configuration)

but that entails some instability leading the estimated coefficients to discard from those of $\bar{B}_{Q}\left(q^{-1}\right)$ in a cyclic manner, as shown in Fig. 3

That leads to employ an overparameterized Youla-Kucera block, with $B_{Q}\left(q^{-1}\right)=\bar{B}_{Q}\left(q^{-1}\right)+D_{p}\left(q^{-1}\right) V\left(q^{-1}\right)$. At first $V\left(q^{-1}\right)$ is chosen to be a scalar (degree 0), which implies that $\operatorname{deg}\left(B_{Q}\left(q^{-1}\right)=2\right.$. If $V \in[0.85,8.93]$, one can check that $\Gamma\left(q^{-1}\right)+\Delta\left(q^{-1}\right) \bar{B}_{Q}\left(q^{-1}\right)+\Delta\left(q^{-1}\right) V\left(q^{-1}\right) D_{p}\left(q^{-1}\right)$ is a stability polynomial, and there exists an infinity of second order polynomials $\hat{B}_{Q}\left(q^{-1}\right)$ allowing for the convergence of $\varepsilon(t+1)$ towards 0 . Consequently the adaptive algorithm will converge: This is exactly what can be observed in Fig. 4.
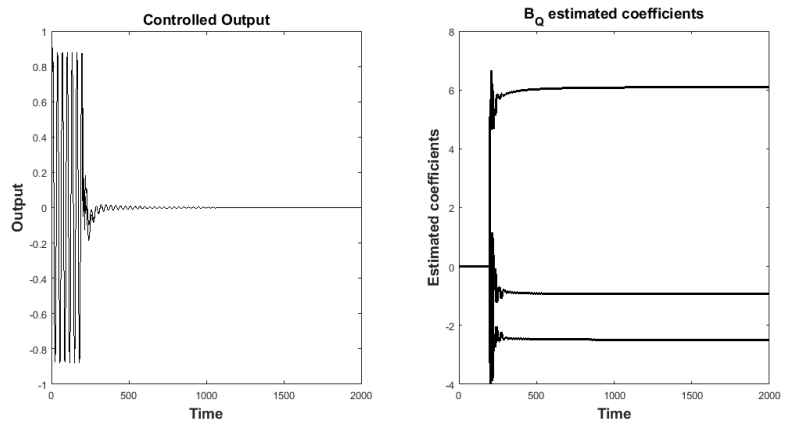

Fig. 4. Adaptive rejection with a second order $\hat{B}_{Q}\left(q^{-1}\right)$, (first uncertainty configuration)

A second uncertainty configuration is now proposed

$$
\begin{gathered}
\Gamma\left(q^{-1}\right)=1-1.4 q^{-1}+0.45 q^{-2} \\
\Delta\left(q^{-1}\right)=0.15 q^{-1}-0.15 q^{-2}
\end{gathered}
$$

The uncertain and nominal systems are displayed in Fig. 5 It can be verified that no scalar $V$ creates the condition for $\left(\Gamma\left(q^{-1}\right)+\Delta\left(q^{-1}\right) \bar{B}_{Q}\left(q^{-1}\right)+\Delta\left(q^{-1}\right) V\left(q^{-1}\right) D_{p}\left(q^{-1}\right)\right)$ to have all its zeros inside the unit circle, and a simulation carried out with a second order polynomial $\hat{B}_{Q}\left(q^{-1}\right)$ does not entail convergence (Fig. 6)

At last we propose to increase the order of $V\left(q^{-1}\right)$, which is set to 1 such that $V\left(q^{-1}\right)=v_{0}+v_{1} q^{-1}$. Thus $\hat{B}_{Q}\left(q^{-1}\right)$ is now a third order polynomial. An evaluation of the closed-loop stability in function of $v_{0}$ et $v_{1}$ over $[-15,+5]$

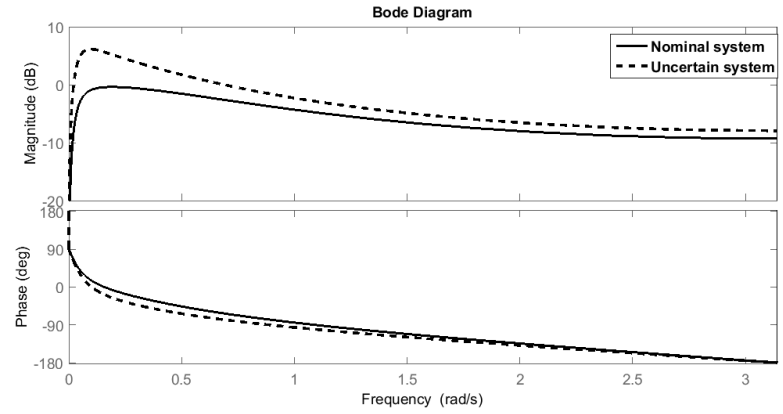

Fig. 5. Uncertain and nominal systems, (second uncertainty configuration)
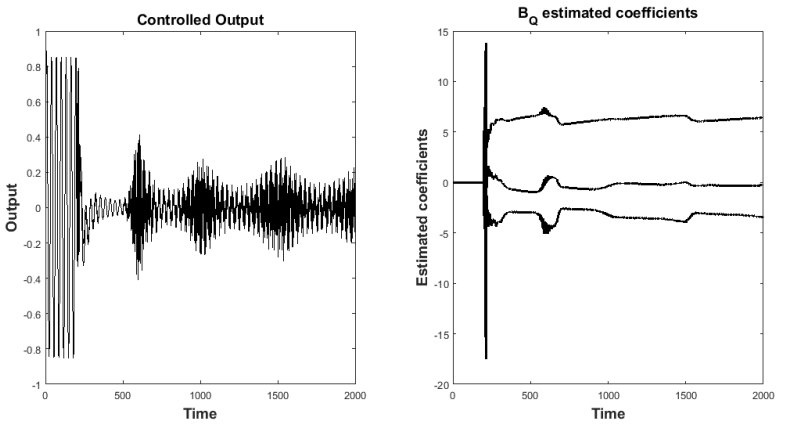

Fig. 6. Adaptive rejection with a second order polynomial $\hat{B}_{Q}\left(q^{-1}\right)$, (second uncertainty configuration).

reveals that a stability area exists as displayed in Fig. 7. Consequently, this algorithm must converge if it includes a third order polynomial $\hat{B}_{Q}\left(q^{-1}\right)$. This is confirmed by simulations as shown in Fig. 8.

\section{CONCLUding REMARKS}

It has been shown that uncertainty linked to the plant model used in the design of an adaptive rejection scheme for the cancellation of unknown narrow band disturbances can lead to instability. The uncertainties have been represented by means of the dual Youla-Kucera parameterization. In order to guarantee stability, two necessary and sufficient conditions must be satisfied at the same time. However, for a minimal order Q-filter in the controller, the said conditions cannot always be met simultaneously. In this case it is necessary to increase the order of the (finite impulse response) Qfilter. We have shown with simulation examples that this overparameterization can create condition for the algorithm to be stable.

\section{REFERENCES}

[1] B. D. O. Anderson, From Youla-Kucera to identification, adaptive and non-linear control, Automatica, vol. 34(12), 1998, pp 1485-1506.

[2] F. Ben Amara, P.T. Kalamba, A.G. Ulsoy, "Adaptive Sinusoidal Disturbance Rejection in Linear Discrete-Time Systems - Part I: Theory", Journal of Dynamic Systems Measurement and Control, vol. 121, pp. 648-654, 1999.

[3] F. Ben Amara, P.T. Kalamba, A.G. Ulsoy, "Adaptive Sinusoidal Disturbance Rejection in Linear Discrete-Time Systems - Part II: Experiments" , Journal of Dynamic Systems Measurement and Control, vol. 121, pp. 655-659, 1999. 


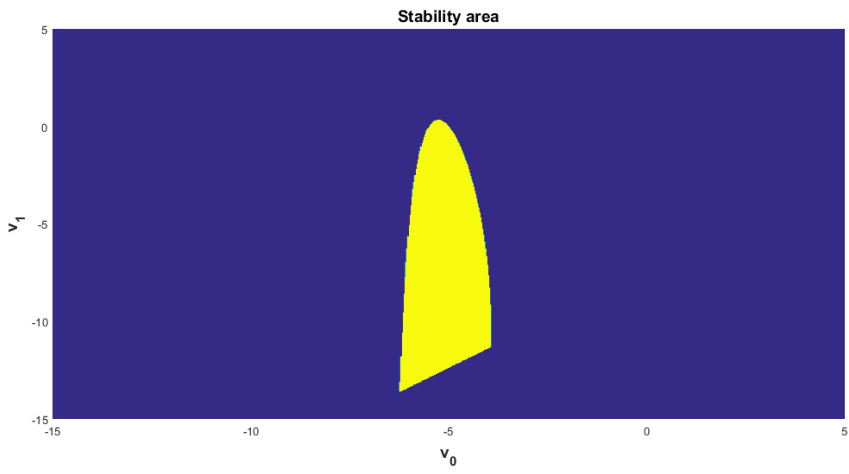

Fig. 7. Stability area (yellow) in function of $v_{0}$ et $v_{1}$ for a third order polynomial $\hat{B}_{Q}\left(q^{-1}\right)$ (second uncertainty configuration)
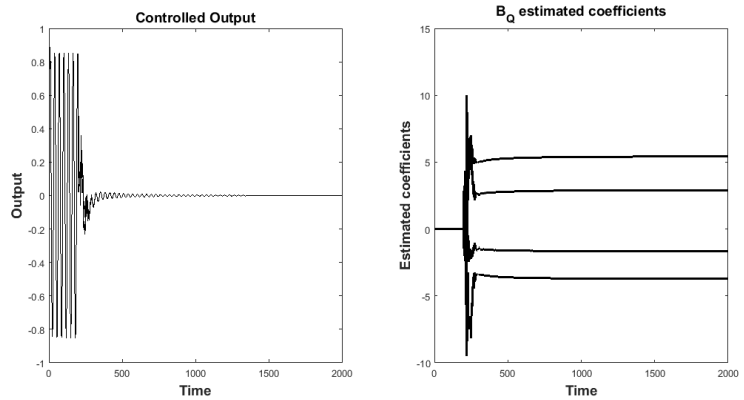

Fig. 8. Adaptive rejection with a third order polynomial $\hat{B}_{Q}\left(q^{-1}\right)$, (second uncertainty configuration)

[4] B.A. Francis, W.M. Wonham, "'The internal model principle of control theory", Automatica, vol. 12, pp. 457-465, 1976.

[5] C.E. Kinney, R.A. de Callafon, "Robust estimation for automatic controller tuning with application to active noise control", in Modelbased Control: Bridging rigorous theory and advanced technology, P.M.J; Van den Hof, C. Scherer, P.S.C Heuberger (Eds), pp.107-124, Springer, 2009.

[6] I.D. Landau, A. Constantinescu, D. Rey, "Adaptive narrow band disturbance rejection applied to an active suspension -an internal model principle approach", Automatica, vol. 41(4), pp.56.-574, 2005.

[7] I.D. Landau, R. Lozano, M. M'Saad, A. Karimi Adaptive control, Springer 2011.

[8] I.D. Landau, R. Melendez, L. Dugard, G. Buche, "Robust and adaptive feedback noise attenuation in ducts", IEEE Transactions on control system technology, vol. 24(12), pp. 1-8, 2017.

[9] T.T.Tay, J.B. Moore, Enhancement of fixed controllers via adaptive$Q$ disturbance estimate feedback, Automatica, vol. 27(1), 1991, pp 39-53.

[10] Ph. Mullhaupt, S. Valentinotti, B. Srinivasan, D. Bonvin, ”Asymptotic rejection of nonvanishing disturbances despite plant-model mismatch", Int; J. Adapt. Control Signal process, vol. 26, pp. 1090-1110, 2012.

[11] P. Van den Hof, R.A. de Callafon, "Multivariable closed-loop identification: From indirect identification to dual Youla Parameterization", proc. 33rd Conference on decision and control, pp. 1397-1402, Kobe, Japan, 1996.

[12] S. Valentinotti, B. Srinivasan, U. Holmberg, D. Bonvin, C. Cannizzaro, M. Rhiel, U. von Stockar, Optimal operation of fed-batch fermentations via adaptive control of overflow metabolite, Control engineering practice, vol. 11, 2003 pp.663-674.

[13] S. Valentinotti, "Adaptive rejection of unstable disturbances: Application to a fed-batch fermentation process", PhD Thesis, Ecole polytechnique fédérale de Lausanne, 2001. 